Corresponding Author:

A/Prof Mike Ford

Institute for Nanoscale Technology, University of Technology, Sydney

PO Box 123, Broadway, NSW 2007, Australia

Email: mike.ford@uts.edu.au

Phone: 61(0)2 95147956

Fax: 61(0)2 95147553

\title{
THE APPLICATION OF GOLD SURFACES AND PARTICLES IN
}

\author{
NANOTECHNOLOGY \\ M.J. FORD, C. MASENS AND M.B. CORTIE \\ Institute for Nanoscale Technology, University of Technology, Sydney, PO Box 123, Broadway,
}

NSW 2007, Australia

Gold is widely used in nanotechnology, for example as a substrate in forming self-assembled monolayers or as nanoparticles for their unique optical and chemical properties. In this paper we give an overview of the properties of gold relevant to its potential application in molecular-scale devices and present some of our recent computational predictions. Density functional calculations of molecular adsorption onto gold surfaces were used to investigate the effect of surface symmetry and identify new linking schemes for self-assembled monolayers. Adsorption energies of methylthiolate $\left(\mathrm{SCH}_{3}\right)$ onto the (111), (100) and (110) surfaces of gold are predicted to be 39.3, 48.4 and $51.1 \mathrm{kcal} / \mathrm{mol}$ respectively and demonstrate that selective functionalisation of the surfaces is possible. Phosphine molecules with at least two hydrogen atoms substituted for methyl groups are predicted to form Au-P surface bonds with energies of about 13-20 kcal/mol.

Keywords: Self-assembled monolayers; Density functional theory; nanotechnology. 


\section{Introduction}

The new field of nanotechnology, which links chemistry, physics, materials science, electrical engineering, and biotechnology, has catalysed a strongly interdisciplinary focus in much of current Science. Metallic gold, either in the form of bulk surfaces or as nanoparticles, is widely used in this emerging technology. ${ }^{1-3}$ This is because of gold's chemical stability, useful surface chemistry, and unique optical properties, which are simultaneously associated with a very convenient range of processing technologies. ${ }^{2,3}$ These features have led to the use of gold in many applications, particularly in biological systems, for example, fluorescent biological, detection of pathogens or proteins, and in blood immunoassays.

In our work we exploit the properties of bulk and nanoscale gold with the aim of producing nanostructured devices which can be switched by light. The conceptual design of such a device would consist of a self-assembled monolayer on a gold substrate with gold nanoparticles attached or embedded into the upper surface of the molecular layer. The gold nanoparticles will absorb energy from the incident light very strongly at a wavelength dependent upon their size and shape, causing a change in the underlying molecular layer. This change may be electronic or conformational in nature and reversible, as for example in a data storage device, or irreversible, for example to lithographically pattern the substrate. Functionality can be tuned through the molecular species while optical response can be varied by controlling the size and shape of the nanoparticles.

This paper presents a particular aspect of our work: first principles density functional calculations for single molecule adsorption on gold. The aim of these studies is two-fold, first to compare the adsorption energetics on the three high symmetry surfaces and second to identify new candidates for linking organic molecules to the substrate. Very few studies exist for the (100) and (110) surfaces or for alternative linking schemes to thiols. From our computational studies it might be possible to devise strategies for controlling the growth of nanoparticles or for preferentially functionalising the surfaces. Before discussing these results we first present, in Section 2, a review of the properties of bulk and nanoscale gold pertinent to our work. Although this is not meant to be 
an exhaustive review it is worthwhile because it draws together themes that, from the literature, appear at first glance to be rather disparate.

\section{Properties and Applications of Gold at the Nanoscale}

Gold does not react with many chemicals. Unlike most other metals its surface does not oxidise easily. Surfaces of gold can be handled in a straight-forward manner without the need for clean, ultra-high vacuum. Preparing ordered, high quality surfaces that are free from contamination is also comparatively easy. Thin films of gold can be prepared by thermal evaporation onto glass, silicon or mica substrates. Thin films prepared by these methods are polycrystalline with grain sizes of approximately 10 to $1000 \mathrm{~nm}$, depending upon substrate temperature and deposition rate. ${ }^{4}$ Gold surfaces that are atomically flat over relatively large areas can be prepared by the method of 'template stripping, ${ }^{5}$ Here, gold is evaporated onto hot mica, then the mica removed by sticky tape, solvent or separated from the gold cryogenically. These large, flat surfaces are important for assembling well-defined molecular nanostructures.

The surface structures of gold are rather more complicated. In common with other closepacked metals the (111) surface is the most thermodynamically stable. The surface energies of the three high index surfaces of gold are reported as $0.05 \mathrm{eV} / \AA^{2}, 0.08 \mathrm{eV} / \AA^{2}$, and $0.100 \mathrm{eV} / \AA^{2}$ for the clean $(111)^{6},(100)^{7}$ and $(110)^{8}$ surfaces respectively. All three low index surfaces undergo reconstruction when clean. ${ }^{9}$ Reconstruction of the clean, densely packed (111) surface is unusual and attributed to relativistic effects in the large electronic core of gold. The $\sqrt{3} \times 22 \mathrm{Au}(111)$ reconstruction is slight, about $4 \%$, and takes the form of compression along the [110] direction with a period of about $63 \AA$, giving alternating domains of fcc and hcp stacking separated by smaller regions with additional bridge site atoms. The overall change in the surface structure is small and has little effect on the electronic properties. ${ }^{7}$ Reconstruction of the clean (110) and (100) surfaces is more dramatic, with a $1 \times 2$ missing row reconstruction for the former and the so-called $5 \times 20$ 
reconstruction for the latter. The $1 \times 2$ missing row reconstruction, where alternate close-packed rows are missing, results in close-packed (111) microfacets. The (100) surface reconstructs to a quasi-hexagonal pattern, similar to $\mathrm{Au}(111)$, with about $25 \%$ more atoms than the open $1 \mathrm{x} 1$ surface.

It is also known that surface reconstructions can be lifted by molecular adsorption. ${ }^{9,10}$ The driving force is the substrate-adsorbate bond itself, or a change in surface charge accompanying adsorption since surface energy depends on surface charge. The latter has been studied quite extensively in electrochemical experiments. ${ }^{8,9,11}$ A $\sqrt{3} \times \sqrt{3} \mathrm{R} 30$ overlayer structure commensurate with the bulk terminated surface for self-assembled monolayers on $\mathrm{Au}(111)$ is commonly observed 2,12,13, indicating that the $\mathrm{Au}(111)$ reconstruction is lifted in the presence of adsorbates. Based upon experimental electrochemical data Santos and Schmickler ${ }^{14}$ have shown that the reconstruction involves a relatively small energy change and will be lifted by surface charging at positive electrode potentials.

For the $\mathrm{Au}(100)$ reconstruction thermodynamic considerations of charging and surface energy predict that lifting of the reconstruction is possible at positive electrode potentials, albeit with a larger energy change compared with $\mathrm{Au}(111) .{ }^{14}$ There is considerable experimental evidence to support this both from electrochemical ${ }^{15,16}$ and adsorption studies. ${ }^{12,17,18}$ Interpretation of the structural data from these experiments is complicated by the fact that a $25 \%$ excess of surface atoms are created in the hexagonal to $1 \mathrm{x} 1$ transition. LEED studies by Dubois et. al. ${ }^{12}$ of alkanethiol adsorption on $\mathrm{Au}(100)$ indicate that the reconstruction is lifted at quite low coverages, well below those required for monolayer formation. Li et. al. ${ }^{18}$ explain their x-ray and atom diffraction measurements for adsorbed n-octadecanethiol monolayers by a structural model where the $\mathrm{Au}$ surface transforms to the unreconstructed $1 \times 1$ with additional rows of $\mathrm{Au}$ atoms on the surface. Similar results are found in a scanning tunneling microscopy (STM) study of ethanethiol adsorption on an $\mathrm{Au}(100)$ electrode $^{16}$ : images taken in air reveal a $25 \%$ coverage of monoatomic high gold islands indicative of the unreconstructed surface. 
The $\mathrm{Au}(110)$ reconstruction is the odd one out. Theory predicts the $1 \times 2$ reconstruction is never lifted by surface charging ${ }^{8}$, but rather the more heavily reconstructed $1 \times 3$ missing row surface becomes more stable at sufficiently negative potential. This prediction is borne out by a number of experimental adsorption studies. ${ }^{19}$

The literature for self-assembled monolayer formation on gold surfaces is extensive. Most of this work exploits the Au-S bond on the (111) surface; for thorough reviews we refer the reader to Love et. al. $2005 .^{2}$ and Ulman. ${ }^{13}$ However, the nature of the thiol-gold bond and assembly process of the monolayer are still unclear. It is accepted that thiol molecules are strongly bound to the gold surface (about $50 \mathrm{kcal} / \mathrm{mol}$ ) through an Au-S bond, however, whether the terminal hydrogen atom is eliminated to produce hydrogen gas (this is most likely for vacuum deposition) or lost through deprotonation is unclear. The structure of ordered thiol SAMs has been discussed above. On the $\mathrm{Au}(111)$ surface the accepted picture is a hexagonal pattern with an intermolecular spacing of $4.98 \AA$ (the $\sqrt{3} \times \sqrt{3} \mathrm{R} 30$ structure) on an unreconstructed surface. The (100) and particularly the (110) surfaces are not so well understood. Based on available evidence it is not unreasonable to assert that the (100) reconstruction is lifted in the presence of the SAM, and the (110) remains reconstructed in the $2 \times 1$ or $3 \times 1$ structures.

Atomistic simulation can provide information regarding the adsorption process and assembly mechanisms at the molecular level. Both full quantum mechanical (QM) DFT ${ }^{20-28}$, Hartree-Fock (HF) and post-HF methods ${ }^{29,30}$ have been applied to accurately describe the adsorbate-substrate chemistry, and force-field molecular mechanics (MM) applied to large overlayer structures in order to probe the thermodynamics. ${ }^{31}$ While DFT methods are an economical way of accurately describing the headgroup-gold interaction they do not describe dispersion-type interactions between the tails of the adsorbed molecules that are responsible for ordering. Post-HF methods can describe these forces but are extremely expensive in terms of computing power, and have been limited to single molecule adsorption on cluster-based models of the gold substrate. In molecular mechanics type simulations the emphasis is placed upon tail-tail 
interactions and ordering dynamics. The application of mixed QM/MM methods, or reliable parameterisation of the Au-S potential ${ }^{22}$ could provide significant gains.

Very few alternatives to sulphur bonds have been proposed in the literature for anchoring organic molecules to gold surfaces. These include carbon in alkyne molecules ${ }^{32}$ and substituted aryl groups ${ }^{33}$ and nitrogen in diisocyanide ${ }^{34}$, and selenium in alkaneselenol ${ }^{35}$ and other organoselenol compounds. ${ }^{36}$ Phosphorous in phosphine ${ }^{37}$ and carbon in phenyactylene ${ }^{38}$ have also been demonstrated as adsorbates on gold nanoparticles.

Gold based SAMs have found recent application in the field of molecular electronics. The idea is to synthesise molecules that act as circuit elements such as diodes and transitors. ${ }^{39}$ This has spawned considerable activity aimed at measuring conduction through various molecules to identify suitable circuit elements. ${ }^{40}$ In these experiments tunneling currents are measured between two gold electrodes spanned by the molecule of choice. The dominant paradigm is that tunneling occurs through the molecule and is influenced by substituents, delocalisation in $\pi$-conjugated molecules or similar effects. Despite considerable progress, few direct comparisons of molecule conductivities exist. A recent article by Moth-Poulson et. $a{ }^{41}{ }^{41}$ compares conductivities for a range of substituted oligo-phenylenevinlylenes; in the results it is difficult to identify any statistically significant change in conductivity for the different molecules. An alternative approach may be to view these devices as simple tunnel barriers with barrier heights that are modified by the presence of a molecule. The molecule alters the surface dipole, and hence work function of the electrodes.

A review of the production and properties of gold nanoparticles has recently been published by Daniel and Astruc. ${ }^{1}$ Most chemical methods for producing colloidal gold nanoparticles rely upon adsorption of molecules onto the nanoparticle in order to stabilise the colloid against aggregation and can have significant effects on particle size and morphology. In some methods the adsorption chemistry results in formation of charged particles which are then stabilised by electrostatic repulsion, while in others the stabilisation is a result of steric effects between the adsorbed molecules. For reasons we will discuss shortly, there is great interest in trying to grow 
nanoparticles of gold with a range of shapes, for example, solid rods, nanoshells, nanocaps, or triangles. In general the spherical form is the most stable, and surfactant-mediated schemes are required to produce the other shapes by wet chemical methods. However, techniques based on polymer sphere lithography offer an alternative route to complex shapes. ${ }^{42}$

Gold nanoparticles have interesting and potentially very useful optical properties. ${ }^{1,43}$ Thin films of gold have a strong resonance at about $500 \mathrm{~nm}$ associated with excitation of a collective motion of the electrons: the so-called bulk plasmon resonance. At wavelengths below this resonance the thin film is more or less transparent, and above it is opaque. This phenomenon has been employed for some time to create optically selective window coatings, and tremendous work has gone into ways to shift the position of the resonance. Gold nanoparticles, and metallic nanoparticles in general exhibit a related phenomena: they absorb incident radiation very strongly at the plasmon resonance. For relatively small spherical gold particles (less than a few hundred nanometers) this resonance, to a first approximation is size independent and occurs at about $520 \mathrm{~nm}$ in water. It is this effect that is responsible for the ruby-red colour of gold colloids. Higher order effects as the particle gets bigger and surface effects as the particle gets smaller shift the resonance to a small degree and attenuate it. As the particle gets larger scattering becomes the dominant effect. $^{44}$ The effects of shape are much more pronounced. For example, it has been predicted that the plasmon resonance in gold nanoshells can be shifted over $300 \mathrm{~nm}$ by simply altering the shell thickness. ${ }^{45}$ Gold nanorods are known experimentally to absorb strongly. Computer simulation also has important role to play here. Analytical solutions to the radiation equations are only possible for the simplest shapes. These have been obtained for solid spheres and closely related shapes using the Mie theory. ${ }^{46}$

\section{Computational Methods}


The $a b$ initio electronic structure calculations of adsorption were performed using the SIESTA software package. ${ }^{47}$ This is a density functional, pseudopotential code with periodic boundary conditions. Rather than using a plane-wave basis, as with most other pseudopotential solid-state codes, atom centred basis sets describe the valence electrons. Counterpoise corrections ${ }^{48}$ are used to estimate the basis set superposition error common in the use of atom centered basis. The generalised-gradient approximation (GGA) to the exchange-correlation functional due to Perdew, Burke and Ernzerhof $(\mathrm{PBE})^{49}$ is used. All calculations are spin unrestricted and employ a Fermi smearing of $25 \mathrm{meV}$ to aid convergence. Norm-conserving pseudopotentials (with a relativistic correction in the case of $\mathrm{Au}$ ) generated according to the scheme of Trouller and Martins ${ }^{50}$ are used. A double-zeta plus single polarisation function is used to represent the valence electrons. These basis sets are strictly localised in real-space, the radial extent of the basis being specified for all atoms by a single energy-shift parameter representing the amount orbital energies are raised due to confinement. We use a value of $0.5 \mathrm{mRy}$ which gives total energies that are converged to better than $0.05 \mathrm{eV}$. Siesta uses a real space grid to calculate potentials, the fineness of this grid is controlled by setting an equivalent plane-wave cutoff, that is the highest energy plane-wave that can be represented on this grid. Final interaction energies were calculated using a mesh cutoff of 180 Ry where total energies are converged to better than $0.15 \mathrm{eV}$

Transferability of the pseudopotentials has been checked by the usual criteria of comparing all-electron and pseudo wavefunctions and their logarithmic derivatives, and inspection of the Fourier transform of the pseudopotential. Gas phase optimisations of the various molecules presented here and of bulk gold have also been performed. In both cases the predicted structures agree to better than 5\% with experimental values. Calculated values for the bulk modulus and cohesive energy of bulk gold give reasonable agreement with experimental values, and are in excellent agreement with other calculations based on plane wave methods. ${ }^{51-53}$

For the adsorption calculations the gold surface is modelled by a slab 5 atomic layers thick in the z-direction and infinite in the $\mathrm{x}-\mathrm{y}$ plane. The calculations are periodic in all three directions 
and so a $30 \AA$ vacuum gap in the z-direction is used to separate the periodically repeating slabs. We have calculated a typical substrate-adsorbate interaction energy as a function of slab thickness and find a variation of less than $3 \mathrm{meV}$ between 5 and 8 layers. Five layers therefore represents a good compromise between accuracy and computational time. The size of the unit cell in the $x-y$ direction is chosen so that the lateral interaction between molecules in adjacent unit cells is small and the distance between them is always greater than about $6 \mathrm{~A}$. For the thiol calculations a $2 \times 2$ cell was used, that is 4 atoms per slab layer, and for the phosphine calculations a $4 \times 4$ unit cell, 16 atoms per layer. A $7 \times 7 \times 1 \mathrm{k}$-point grid (28 k-points) is used to sample the reciprocal space unit cell for the thiol calculations and a $3 \times 3 \times 1$ (6 k-points) for the phosphine calculations. Both conditions give total energies that are converged to better than $0.1 \mathrm{eV}$.

Different methods have been used to find the optimum adsorption site for the thiol calculations and phosphine calculations. In all cases the isolated molecule and isolated slab were initially relaxed using a conjugate gradient (CG) optimisation. For methylthiolate the radical + surface layer of slab were then relaxed starting from likely adsorption sites. For the phosphine calculations the optimised molecule was scanned across the optimised surface in a series of singlepoint calculations to locate the minimum energy site. Convergence criteria on the forces for the CG optimisations were set at a relatively low level of $0.04 \mathrm{eV} / \mathrm{Ang}$. Even so, these criteria result in a well-optimised bond length with interaction energies being essentially the same when the tolerance is tightened to $0.01 \mathrm{eV} / \mathrm{Ang}$.

\section{Results and Discussion}

In this section we present our first principles DFT calculations for adsorption onto gold surfaces. The effects of surface symmetry on the adsorption energetics are first explored through adsorption of the methylthiolate radical onto the three low index surfaces of gold. Although this work has been presented in detail elsewhere ${ }^{51,52}$, we summarise the results here as it complements the 
adsorption studies of phosphine molecules and radicals onto $\mathrm{Au}(111)$. Here, we investigate first whether phosphine can form a link to the gold surface. By successively replacing $\mathrm{H}$ atoms with methyl groups we can assess substituent effects on adsorption.

In all cases the (111) and (100) surfaces are assumed to be unreconstructed and the (110) reconstructed into the $2 \times 1$ missing row. From the discussion in Section 2 this is reasonable based upon the available evidence. The gold slabs were constructed using the calculated optimum lattice parameter for bulk gold of $4.18 \AA$. The experimental value is $4.079 \AA^{54}$. Slight overestimation of the lattice parameter in GGA calculations is expected. The slabs were then allowed to relax in a direction normal to the surface, that is interlayer spacings are optimised but the surface does not reconstruct. For the (111) and (100) surfaces there is a slight contraction of the outermost layer by $0.5 \%$ and $1.2 \%$ respectively. Gronbeck et $a .^{24}$ report a contraction in the outermost layer of $\mathrm{Au}(111)$ of about $1 \%$, and Molina and $\mathrm{Hammer}^{26}$ about $0.5 \%$ contraction. Calculated relaxation of the 5 layer (110) surface is dramatic, in order to discount slab thickness effects we performed a relaxation of a 10 layer slab with similar results. The outermost atoms contract by about $18 \%$. This is not surprising considering these atoms do not form a complete layer in the missing row reconstruction. The next layer down expands by about $3 \%$.

\subsection{Effect of Surface Symmetry on Adsorption}

Starting positions for the geometry optimisations of methylthiolate on the three low index faces of gold are shown in Figure 1. In these calculations the $\mathrm{SCH}_{3}$ radical is adsorbed to the surface, and both the radical and surface layer gold atoms are allowed to relax. The reconstruction of the (110) surface is not obvious in plane view; starting positions $\mathrm{d}$ and $\mathrm{h}$ are directly over the missing row of atoms.

Interaction energies and bond lengths for the energetically most favourable site on each surface are given in Table 1. They are calculated by taking the difference between the energy of the optimised slab+radical and sum of isolated slab+isolated radical. It is, in a sense, a non-adiabatic 
interaction energy since energies of the isolated components are calculated by ghosting the other component without relaxation. The values are therefore BSSE corrected, uncorrected interactions are about $10 \%$ larger. The geometry of the adsorbed radical is very close to the gas phase molecule, and relaxation of the outer layer of the surface is less than $5 \%$ upon adsorption. Optimum adsorption geometries for the three surfaces are shown in Figure 2.

For the (111) surface the optimum site is between the fcc hollow and bridge sites, with the sulphur atom positioned $0.3 \AA$ from the bridge site and the methyl tail group tilted at $42^{\circ}$ from the surface normal over the bridge site. Optimum adsorption sites are given in Figure 2. Starting position $\mathrm{b}$ in Figure 1 optimised to this final geometry. The $2 \times 2$ unit cell used corresponds to a coverage of 0.75 times that of the $\sqrt{3} \times \sqrt{3} \mathrm{R} 30$ overlayer. Bond lengths to the two bridging Au atoms are $2.5 \AA$, and to the third hollow site atom 2.9 $\AA$. Based upon Mulliken overlap populations the adsorbate-substrate bond is covalent in character and is between the $\mathrm{S}$ atom and the two Au bridge atoms. The overlap population is $0.21 \mathrm{e}$ for each bond, overlap with the third hollow site atom is 0.08e. Our predicted optimum absorption geometry and energy is in very good agreement with the results of Yourdshahyan and Rappe ${ }^{28}$ using a plane-wave DFT method. It is also consistent with the calculations of Gottschalk et. al. ${ }^{55}$ and Hayashi et. $a l .{ }^{25}$, although the latter gives an adsorption energy relative to dimethyl-disulphide. This is an important result as it indicates that our correction for BSSE is reliable. The counterpoise method is only an approximate way to correct for BSSE, although for the strong covalent bonds here and with the relatively small double-zeta basis set employed one might expect it to yield a reliable estimate. Other values for the adsorption energy and optimum site can be found in the literature, some of these however, model the gold substrate using a 2 layer cluster and might therefore be expected to give different results. ${ }^{21,22,30}$ Plane-wave DFT calculations by Andreoni et. al. $^{20}$ and Vargas et. $a .^{27}$ identify the fcc and bridge sites, respectively, as optimum. The former work quotes an adsorption energy of $55 \mathrm{kcal} / \mathrm{mol}$.

Optimisations starting from positions $\mathrm{c}$ and $\mathrm{d}$, bridge and hcp respectively, give final geometries where the $\mathrm{S}$ atom has moved only very slightly towards the hcp and bridge sites 
respectively. The adsorption energies are $38.9 \mathrm{kcal} / \mathrm{mol}$ and $34.5 \mathrm{kcal} / \mathrm{mol} \mathrm{respectively.} \mathrm{The} \mathrm{atop}$ starting site, a, optimises to a final geometry where the radical is adsorbed in the hcp site.

Relaxation starting from the bridge site yields the optimum adsorption site for the (100) surface, with the S atom almost half-way between the bridge and four-fold hollow sites, as shown in Figure 2. The methyl tail group is tilted at $49^{\circ}$ to the surface normal. Again, the bond is quite covalent in nature and two-fold coordination with the surface is preferred. Overlap populations between the $\mathrm{S}$ atom and nearest two bridge site $\mathrm{Au}$ atoms are $0.17 \mathrm{e}$ and the corresponding bond lengths are $2.5 \AA$. The four-fold hollow is a local minimum with adsorption energy of 43.5 $\mathrm{kcal} / \mathrm{mol}$, while the atop site is a saddle point and relaxes to the four-fold hollow.

A large number of starting sites were used for the reconstructed (110) surface due to its lower symmetry. However, all 8 starting geometries essentially relax to only two final adsorption sites, slightly away from the bridge site of the top most atomic row (step-edge, or b in Figure 1) and four-fold hollow at the missing row (h in Figure 1). The step-edge site with the radical tilted $20^{\circ}$ away from the surface normal over the bridge site gives the highest adsorption energy (Figure 2). Bond lengths between the S atom and two nearest $\mathrm{Au}$ atoms are $2.5 \AA$ and corresponding overlap populations are $0.16 \mathrm{e}$. The different starting positions do, however, give different final tilt angles in the relaxed geometries, indicating that there are a number of local minima with respect to rotating the radical. Nonetheless, all the final step-edge geometries give adsorption energies that are more favourable than the final four-fold hollow geometries.

\subsection{Adsorption of Phosphines}

Adsorption energetics of the phosphine molecules, $\mathrm{PH}_{3}, \mathrm{PH}_{2} \mathrm{CH}_{3}, \mathrm{PH}\left(\mathrm{CH}_{3}\right)_{2}$ and $\mathrm{P}\left(\mathrm{CH}_{3}\right)_{3}$, and the radicals $\mathrm{PHCH}_{3}$ and $\mathrm{P}\left(\mathrm{CH}_{3}\right)_{2}$ on the $\mathrm{Au}(111)$ surface were determined by calculating the potential energy surface for the rigid adsorbate above the surface ${ }^{52}$. The molecule or radical is oriented with the phosphorous atom pointing towards the surface and the tail groups away from the surface. Previous XPS studies indicate this is the most likely adsorption geometry. ${ }^{56}$ The gas phase 
molecule, or radical and $\mathrm{Au}(111)$ were first geometry optimised. The total DFT energy was then calculated for the molecule (or radical) at a fixed height of $2.75 \AA$ on a grid of $10 x 10$ points above a representative section of the (111) surface. At the lowest energy site of this potential energy surface the molecule was rotated around the surface normal through 360 in 60 steps to identify the optimum orientation. Finally, the total energy was calculated at 20 points with the height of the molecule ranging from 1.5 to $3.5 \AA$ above the surface in order to determine the optimum height. Adsorption energies at the lowest energy position where then calculated by ghosting successively the adsorbate and gold slab. As in the previous section, non-adiabatic, BSSE corrected interaction energies are determined.

For the $\mathrm{PH}_{3}, \mathrm{PH}_{2} \mathrm{CH}_{3}$ molecules the minimum of the potential energy surface occurs at the hollow sites, whereas for the $\mathrm{PH}\left(\mathrm{CH}_{3}\right)_{2}$ and $\mathrm{P}\left(\mathrm{CH}_{3}\right)_{3}$ molecules and $\mathrm{PHCH}_{3}$ and $\mathrm{P}\left(\mathrm{CH}_{3}\right)_{2}$ radical the minimum is at, or close to, the atop site. Phosphine and methyl-phosphine are found to be unbound, whereas all other adsorbates considered were found to be bound with respect to the isolated molecule or radical and gold slab. The final interaction energies are given in table 2 .

The dimethyl-phosphine and trimethyl-phosphine molecules will adsorb strongly to the gold surface. Previous temperature programmed desorption (TPD) studies on monolayers of dimethylphenylphosphine claim stability up to $500 \mathrm{~K}$ in ultrahigh vacuum, and XPS studies on tricyclohexylphosphine indicate stability of the monolayer adsorbed on gold ${ }^{56}$. The adsorption energies for these two molecules given in Table 2 are essentially the heat of reaction of the adsorbate-substrate. Thiol molecules, by contrast only adsorb strongly once the terminal S-H bond has been cleaved. Although the adsorption energy for methylthiolate on Au(111) is twice as large as the phosphines, the overall reaction energy for methanethiol adsorption is only a few $\mathrm{kcal} / \mathrm{mol}^{2}$ The adsorption heights reported in Table 2 are in reasonable agreement with the crystal structure of a triphenylphosphine gold complex where the Au-P bond length is measured to be $2.3 \AA .^{57}$

The phosphorous atom bonds to a single gold surface atom as indicated by the Mulliken overlap populations. The Au-P overlaps for dimethyl-phosphine and trimethyl-phosphine are 0.15 
and 0.21 respectively. As might be expected the overlap increases with the bond strength, the adsorption height also decreases as the bond becomes stronger. Adsorption of the phosphine molecule onto the surface is clearly stabilised by substitution of methyl groups. Comparing Mulliken charges for gas phase and surface bound trimethylphosphine, the charge of the $\mathrm{P}$ atom becomes $1.4 \mathrm{e}$ more positive upon adsorption with charge being lost form the $3 \mathrm{~s}$ and $3 \mathrm{p}$ orbitals, conversely the three $\mathrm{C}$ atoms become more negative by a total of $1.2 \mathrm{e}$ with charge donated into the $2 \mathrm{~s}$ and $2 \mathrm{p}$ orbitals. The gold surface takes up the remaining $0.2 \mathrm{e}$ of negative charge which appears to be distributed over the gold atoms. This can also be seen in the electron density and density difference maps for dimethyl-phosphine shown in Figure 3. The P atom has become more negative in the density difference map, while the electron density has increased in the C-P bonds. There also appears to be a region of charge sharing between the $\mathrm{P}$ atom and nearest $\mathrm{Au}$ atom. A possible way to rationalise this result and explain stabilisation by methyl groups is that phosphorous donates electrons into the gold surface, presumably from the lone pair, and the gold back-donates into the phosphorous-carbon bond. As more methyl groups are added back donation stabilises the adsorbate-substrate bond more effectively. Similar arguments have previously been proposed to explain the stability of $\mathrm{F}$ or $\mathrm{Cl}$ substituted phosphine ligands. ${ }^{58}$

Comparing the adsorption energies for the two radicals, $\mathrm{PHCH}_{3}$ and $\mathrm{P}\left(\mathrm{CH}_{3}\right)_{2}$, with the corresponding molecules we see that removal of the hydrogen atom stabilises binding of the single methyl substituted phosphine. The adsorption energy increases for the dimethyl substituted case upon removal of the hydrogen, but not as dramatically as might be expected, by about $5 \mathrm{kcal} / \mathrm{mol}$. The Au-P overlap populations for the two radicals are the same, 0.25 and adsorption heights less than either of the bound molecules. Electron charge density and density difference maps for $\mathrm{P}\left(\mathrm{CH}_{3}\right)_{2}$ are shown in Figure 4 in a plane normal to the surface containing the $\mathrm{P}$ atom. The two $\mathrm{C}$ atom are out of the plane of these images. The density difference again shows charge located between the $\mathrm{P}$ atom and single $\mathrm{Au}$ surface atom similar to the corresponding dimethyl-phosphine molecule in Figure 3. Adsorption of the radical species is most likely still through the lone pair 
electrons, the additional unpaired electron from the hydrogen bond may provide additional electron density to stabilise this bond but does not participate directly. This could potentially provide a pathway for functionalising the surface, where the molecule is adsorbed to the surface then the hydrogen removed to create a surface bound species that is chemically reactive.

\section{Conclusions}

The adsorption energetics of methylthiolate on three high index surfaces of gold have been calculated using density functional theory. For the (111) surface we find that the energetically most favourable site is the sulphur atom positioned between the fcc and bridge sites and the methyl tail group tilted at $42^{\circ}$ over the bridge site. The adsorption energy is $-39.3 \mathrm{kcal} / \mathrm{mol}$ with the sulphur atom coordinated to two of the surface gold atoms. Methylthiolate is more strongly adsorbed to the two open surfaces (100) and (110), with energies of $-48.4 \mathrm{kcal} / \mathrm{mol}$ and $-51.1 \mathrm{kcal} / \mathrm{mol}$ respectively. This result is not surprising given the higher coordination of gold atoms on the (111) surface compared to either the (110) or (100) surfaces. In both these cases the sulphur atom is also coordinated with two surface gold atoms. A site between the bridge and four-fold hollow is energetically most favourable for the (100) surface, and just off the step-edge towards a bridge site for the (110) surface. For all three surfaces the gold-sulphur bond lengths are $2.5 \AA$.

These results show that it is, in principle, energetically possible to selectively adsorb to the (111) or (100) / (110) surfaces. The predicted difference in adsorption energy between the (100) and (110) surfaces is probably at about the level of reliability of the present calculations. Whether these two surfaces can be selectively functionalised is, therefore, not so clear. Selective adsorption onto the different faces presented by a gold nanoparticle would provide a means for controlling particle growth. While this is the case for the (111) surfaces versus either of the other two surfaces, reconstruction of the (110) surface in the presence of an absorbate presumably reduces the adsorption energy relative to the unreconstructed surface thereby giving an adsorption energy close 
to the (100) surface. If both were unreconstructed one might expect different adsorption energies for these two surfaces also.

Calculations for the adsorption of the phosphine molecule and methyl substituted phosphines predict that adsorption is energetically possible provided at least two of the hydrogen atoms have been substituted. The adsorption energies of the di- and trimethyl-phosphine are -13.5 and $-20.6 \mathrm{kcal} / \mathrm{mol}$. Removal of one hydrogen atom from these molecules to create a radical increases the binding energy. Both the $\mathrm{PHCH}_{3}$ radical and $\mathrm{P}(\mathrm{CH})_{2}$ radicals are bound by -14.1 $\mathrm{kcal} / \mathrm{mol}$ and $-18.5 \mathrm{kcal} / \mathrm{mol}$. In all cases the molecule or radical is bound through the phosphorous atom with the tail groups oriented away from the surface. The atop site, or close to it, is most favourable in all cases. These results suggest that phosphorous binds through its lone electron pair to give a tetrahedral coordination with the three tail groups and single surface gold atom. Substitution of methyl groups stabilises the bond through electron donation onto the phosphorous. The unpaired electron in the radical, left by removal of a hydrogen atom, does not participate directly in the bond, but does appear to stabilise the bond further, presumably through additional electron density on the phosphorous atom.

Phosphorous containing molecules would appear to provide an alternative to thiol molecules for forming surfaces layers on gold. In addition, they bind relatively strongly to gold even as molecules (as opposed to thiolate radicals in the case of sulphur). 


\section{Acknowledgements}

This work was supported by the University of Technology, Sydney and the Australian Research Council. High performance computing facilities were provided under the merit allocation schemes of the Australian Centre for Advanced Computing and Communications (in New South Wales) and the National Facility at the Australian Partnership for Advanced Computing. The authors acknowledge Dr Helen Dorsett for her insight into surface chemistry.

\section{References}

1. $\quad$ M.-C. Daniel and D. Astruc, Chem. Rev. 104, 293 (2004).

2. J. C. Love, E. L. A, J. K. Kriebel, G. Nuzzoet al., Chem. Rev. 105, 1103 (2005).

3. M. B. Cortie, Gold Bulletin 37, 12 (2004).

4. C. E. D. Chidsey, D. N. Loiacono, T. Sleator, and S. Nakahara, Surf. Sci. 200, 45 (1988); A. Putnam, B. L. Blackford, M. H. Jericho, and M. O. Watanabe, Surf. Sci. 217, 276 (1989); J. A. DeRose, T. Thundat, L. A. Nagahara, and S. M. Lindsay, Surf. Sci. 256, 102 (1991).

5. M. Hegner, P. Wagner, and G. Semenza, Surf. Sci. 291, 39 (1993); D. Losic, J. G. Shapter, and J. J. Gooding, Aust. J. Chem. 54, 643 (2001).

6. Z. Crljen, P. Lazic, D. Sokcevic, and R. Brako, Phys. Rev. B: Condens. Matter 68, 195411 (2003).

7. N. Takeuchi, C. T. Chan, and K. M. Ho, Phys. Rev. B: Condens. Matter 43 (17), 13899 (1991).

8. A. Y. Lozovoi and A. Alavi, Phys. Rev. B: Condens. Matter 68, 245416 (2003).

9. D. M. Kolb, Prog. Surf. Sci. 51 (2), 109 (1996).

10. G. A. Somorjai and M. A. Van Hove, Prog. Surf. Sci. 30, 201 (1989).

11. W. Schmickler, Chem. Rev. 96, 3177 (1996).

12. L. H. Dubois, R. Zegarski, and G. Nuzzo, J. Chem. Phys. 98 (1), 678 (1993).

13. A. Ulman, Chem. Rev. 96, 1533 (1996).

14. E. Santos and W. Schmickler, Chem. Phys. Lett. 400, 26 (2004).

15. M. Labayen and O. M. Magnussen, Surf. Sci. 573, 128 (2004); X. Gao, A. Hamelin, and M. J. Weaver, Phys. Rev. Lett. 67 (5), 618 (1991).

16. M. Schweizer, H. Hagenstrom, and D. M. Kolb, Surf. Sci. 490, L627 (2001).

17. E. D. L. Rienks, G. P. van Berkel, J. W. Bakker, and B. E. Nieuwenhuys, Surf. Sci. 571, 187 (2004).

18. J. Li, K. S. Liang, N. Camillone, T. Y. B. Leunget al., J. Chem. Phys. 102 (12), 5012 (1995).

19. P. Guaino, D. Carty, G. Hughes, O. McDonaldet al., Appl. Phys. Lett. 85 (14), 2777 (2004);

A. Cossaro, D. Cvetko, G. Bavdek, L. Floreanoet al., J. Phys. Chem. B 108, 14671 (2004);

X. Zhao, H. Yan, R. G. Zhao, and W. S. Yang, Langmuir 18, 3910 (2002).

20. W. Andreoni, A. Curioni, and H. Gronbeck, Int. J. Quantum Chem. 80 (4-5), 598 (2000).

21. K. M. Beardmore, J. D. Kress, A. R. Bishop, and N. Grøbech-Jensen, Synth. Met. 84, 317 (1997).

22. K. M. Beardmore, J. D. Kress, N. Gronbechjensen, and A. R. Bishop, Chem. Phys. Lett. 286 (1-2), 40 (1998). 
23. A. Bilic, J. R. Reimers, and N. S. Hush, J. Chem. Phys. 122 (9), 094708 (2005); C.

Majumder, T. M. Briere, H. Mizuseki, and Y. Kawazoe, J. Chem. Phys. 117 (6), 2819 (2002); Y. Yourdshahyan, H. K. Zhang, and A. M. Rappe, Phys. Rev. B 63 (081405(R)), 081405 (2001).

24. H. Gronbeck, A. Curioni, and W. Andreoni, J. Am. Chem. Soc. 122, 3839 (2000).

25. T. Hayashi, Y. Morikawa, and H. Nozoye, J. Chem. Phys. 114 (17), 7615 (2001).

26. L. M. Molina and B. Hammer, Chem. Phys. Lett. 360, 264 (2002).

27. M. C. Vargas, P. Giannozzi, A. Selloni, and G. Scolee, J. Phys. Chem. B 105, 9509 (2001).

28. Y. Yourdshahyan and A. M. Rappe, J. Chem. Phys. 117 (2), 825 (2002).

29. H. Sellers, Surf. Sci. 294, 99 (1993).

30. H. Sellers, A. Ulman, and Y. Shnidman, J. Am. Chem. Soc. 115, 9389 (1993).

31. S. Jiang, Mol. Phys. 100 (14), 2261 (2002); N. Gronbech-Jensen, A. N. Parikh, K. M. Beardmore, and R. C. Desai, Langmuir 19, 1474 (2003); Y. Leng, D. J. Keffer, and P. T. Cummings, J. Phys. Chem. B 107, 11940 (2003).

32. H. Feilchenfeld and M. J. Weaver, J. Phys. Chem. 93, 4276 (1989).

33. M.-C. Bernard, A. Chausse, E. Cabet-Deliry, M. M. Chehimiet al., Chem. Mater. 15, 3450 (2003); A. Laforgue, T. Addou, and D. Belanger, Langmuir, In Press (2005); G. Liu, J. Liu, T. Bocking, P. K. Eggerset al., Chem. Phys., In Press (2005).

34. S. A. Swanson, R. McClain, K. S. Lovejoy, N. B. Alamdariet al., Langmuir 21, 5034 (2005).

35. L. V. Protsailo, W. R. Fawcett, D. Russell, and R. L. Meyer, Langmuir 18, 9342 (2002).

36. S. W. Han and K. Kim, J. Colloid Interface Sci. 240, 492 (2001).

37. W. W. Weare, S. M. Reed, M. G. Warner, and J. E. Hutchison, J. Am. Chem. Soc. 122, $12890(2000)$.

38. S.-W. Joo and K. Kim, J. Raman Spectrosc. 35, 549 (2004).

39. A. Aviram and M. A. Ratner, Chem. Phys. Lett. 29, 277 (1974).

40. D. K. James and J. M. Tour, Chem. Mater. 16, 4423 (2004).

41. K. Moth-Poulsen, L. Patrone, N. Stuhr-Hansen, J. B. Christensenet al., Nano Letters 5 (4), 783 (2005).

42. J. Liu, A. I. Maaroof, L. Wieczorek, and M. B. Cortie, Advanced Materials 17, 1276 (2005).

43. K. L. Kelly, E. Coronado, L. L. Zhao, and G. C. Schatz, J. Phys. Chem. B 107 (668-677) (2003).

44. C. F. Bohren and D. R. Huffmann, Absorption and scattering of light by small particles. (Wiley, New York, 1998).

45. S. J. Oldenberg, R. D. Averitt, S. L. Westcott, and N. J. Halas, Chem. Phys. Lett. 288, 243 (1998).

46. G. Mie, Ann. Phys. 25, 377 (1908).

47. P. Ordejon, E. Artacho, and J. M. Soler, Phys. Rev. B: Condens. Matter 53, R10441 (1996);

J. M. Soler, E. Artacho, J. D. Gale, A. Garciaet al., J. Phys.: Condens. Matter 14, 2745 (2002).

48. S. B. Boys and F. Bernardi, Mol. Phys. 19, 533 (1970).

49. J. P. Perdew, K. Burke, and M. Ernzerhof, Phys. Rev. Lett. 77 (18), 3865 (1996).

50. $\quad$ N. Troullier and J. L. Martins, Phys. Rev. B 43 (3), 1993 (1991).

51. C. Masens, M. J. Ford, and M. B. Cortie, Surf. Sci. 580 (19-19) (2005).

52. C. Masens, PhD Dissertation, University of Technology, 2004.

53. B. Soule de Bas, M. J. Ford, and M. B. Cortie, J. Mol. Struct: THEOCHEM 686, 193 (2004).

54. A. Khein, D. J. Singh, and C. J. Umrigar, Phys. Rev. B 51, 4105 (1995).

55. J. Gottschalk and B. Hammer, J. Chem. Phys. 116, 784 (2002).

56. K. Uvdal, I. Persson, and B. Liedberg, Langmuir 11, 1252 (1995). 
57. S. A. Larkin, J. A. Krause Bauer, V. E. Konoplev, V. P. Dyadchenkoet al., Acta Crystallogr., Sect. C: Cryst. Struct. Commun. C60, m440 (2004).

58. S.-X. Xiao, W. C. Trogler, D. E. Ellis, and Z. Berkovitch-Yellin, J. Am. Chem. Soc. 105, 7033 (1983). 


\section{Figure Captions.}

Figure 1. Starting positions for geometry optimisations on the three gold surfaces.

Figure 2. Optimum adsorption geometries for the three gold surfaces. Third column of atoms from the left is the missing row for the (110) surface.

Figure 3. Electron charge density and charge density difference for $\mathrm{HP}\left(\mathrm{CH}_{3}\right)_{2}$. Both images are viewed in a plane normal to the surface containing the $\mathrm{P}$ atom and two $\mathrm{C}$ atoms.

Figure 4. Electron charge density and charge density difference for the $\mathrm{P}\left(\mathrm{CH}_{3}\right)_{2}$ radical. Both images are viewed in a plane normal to the surface containing the $\mathrm{P}$ atom. The two $\mathrm{C}$ atom are out of the plane of these images. 
Table 1. Interaction energies for the three low index surfaces at the optimum site

\begin{tabular}{lcc}
\hline Surface / Site & $\begin{array}{c}\text { Au-S } \\
\text { Bond length }(\AA)\end{array}$ & $\begin{array}{c}\text { Energy } \\
(\mathrm{kcal} / \mathrm{mol})\end{array}$ \\
\hline $\begin{array}{l}(111) / \text { fcc-bridge } \\
(100) / \text { four-fold }\end{array}$ & 2.5 & -39.3 \\
$\begin{array}{l}\text { hollow-bridge } \\
(110) / \text { step-edge }\end{array}$ & 2.5 & -48.4 \\
\hline
\end{tabular}


Table 2. Adsorption energies and heights for phosphine molecules and radicals on $\mathrm{Au}(111)$.

\begin{tabular}{lcc}
\hline \multicolumn{1}{c}{ Adsorbate } & Height $(\AA)$ & Energy $(\mathrm{kcal} / \mathrm{mol})$ \\
\hline $\mathrm{PH}_{3}$ & - & - \\
$\mathrm{PH}_{2} \mathrm{CH}_{3}$ & - & - \\
$\mathrm{PH}\left(\mathrm{CH}_{3}\right)_{2}$ & 2.74 & -13.5 \\
$\mathrm{P}\left(\mathrm{CH}_{3}\right)_{3}$ & 2.53 & -20.6 \\
$\mathrm{PHCH}_{3}$ & 2.32 & -14.1 \\
$\mathrm{P}\left(\mathrm{CH}_{3}\right)_{2}$ & 2.35 & -18.5 \\
\hline
\end{tabular}

